Am. J. Trop. Med. Hyg., 71(Suppl 2), 2004, pp. 174-178

Copyright $@ 2004$ by The American Society of Tropical Medicine and Hygiene

\title{
DO MALARIA CONTROL INTERVENTIONS REACH THE POOR? A VIEW THROUGH THE EQUITY LENS
}

\author{
LAWRENCE M. BARAT, NATASHA PALMER, SUPROTIK BASU, EVE WORRALL, KARA HANSON, AND ANNE MILLS \\ Human Development, Africa Region, World Bank, Washington, District of Columbia; Malaria Branch, Division of Parasitic \\ Diseases, Centers for Disease Control and Prevention, Atlanta, Georgia; Health Economics and Financing Programme, London \\ School of Hygiene and Tropical Medicine, London, United Kingdom; Roll Back Malaria Secretariat, World Health Organization, \\ Geneva, Switzerland
}

\begin{abstract}
Malaria, more than any other disease of major public health importance in developing countries, disproportionately affects poor people, with $58 \%$ of malaria cases occurring in the poorest $20 \%$ of the world's population. If malaria control interventions are to achieve their desired impact, they must reach the poorest segments of the populations of developing countries. Unfortunately, a growing body of evidence from benefit-incidence analyses has demonstrated that many public health interventions that were designed to aid the poor are not reaching their intended target. For example, the poorest $20 \%$ of people in selected developing countries were as much as 2.5 times less likely to receive basic public health services as the least-poor $20 \%$. In the field of malaria control, a small number of studies have begun to shed light on differences by wealth status of malaria burden and of access to treatment and prevention services. These early studies found no clear difference in fever incidence based on wealth status, but did show significant disparities in both the consequences of malaria and in the use of malaria prevention and treatment services. Further study is needed to elucidate the underlying factors that contribute to these disparities, and to examine possible inequities related to gender, social class, or other factors. To achieve impact and overcome such inequities, malaria control efforts must begin to incorporate approaches relevant to equity in program design, implementation, and monitoring and evaluation.
\end{abstract}

\section{MALARIA: A DISEASE OF POVERTY}

Malaria is confined almost exclusively to developing countries, particularly in sub-Saharan Africa and southern Asia, the poorest regions of the world. Gwatkin and Guillot demonstrated that $58 \%$ of the malaria deaths occurred in the poorest $20 \%$ of the world's population, a higher percentage than for any other disease of major public health importance (Table 1). ${ }^{1}$

Within these poor countries, malaria disproportionately affects the poorest of the poor populations. Reaching the poorest of the poor with malaria control interventions poses great challenges, not solely because of financial barriers to accessing care and prevention services. The poorest populations in developing countries often live in the most remote areas and are socially or culturally marginalized.

In the global development community, concerns that public health interventions may not be reaching poor and marginalized populations have led investigators to examine the differences in the burden of disease and the coverage and impact of public health interventions among persons with differing socioeconomic status (SES). One of the primary tools in this line of investigation has been benefit-incidence analysis, in which the disease burden and the use of health care services and the government subsidies included in them are measured across different socioeconomic groups. ${ }^{2}$

The results of early studies have begun to demonstrate striking disparities in the use of public health services by the poorest when compared with less poor populations. Although these services are often intended to reach the poor, the poorest groups have been shown to be least likely to receive the benefits of those services. For example, analyses of Demographic and Health Survey (DHS) data have documented that the wealthiest $20 \%$ of the population of 44 developing countries were 1.25 to more than 2.5 times more likely than the poorest $20 \%$ to receive key public health services, including treatment of diarrhea, childhood immunization, and antenatal care (Figure 1) (Gwatkin DR, unpublished data).

Because the negative consequences of malaria fall most heavily on the poorest segments of the population, such disparities in the use of public health services must be of particular concern to the malaria control community. If extra efforts are not made to reach the poorest of the poor with effective malaria control interventions, it is very likely that the Roll Back Malaria (RBM) target of reducing the global malaria burden by $50 \%$ by the year 2010 will not be reached. Interventions, therefore, must be designed to ensure that a large percentage of the most poor are using effective treatment, insecticide-treated bed nets (ITNs), and other essential malaria control interventions. This can only be accomplished with a broader understanding of what types of approaches are best at reaching the poor and what barriers limit access and use of essential malaria control services.

\section{DEFINITIONS AND MEASUREMENTS}

A working definition for inequities in health are differences that are "not only unnecessary and avoidable, but in addition, are considered unfair and unjust."3 Although much of the work on inequities, to date, has focused on SES, other factors that might lead to inequities include sex, ethnicity, and social class. Although there are quantitative measures for SES and sex, measurement of factors like social status cannot be easily quantified. Qualitative research can be used to explore the role of these less tangible factors and thus help to identify underlying reasons why people do or do not use particular services.

Even with SES, there is controversy over how it should be measured. Because much of the commerce in developing countries is not cash-based and occurs outside the formal economy, measurement of income or consumption is difficult and these are unreliable measures of SES. Researchers, therefore, have used so-called "asset indices" to measure household well-being. Such indices are constructed by assessing household ownership of specific items (e.g., radios, bicycles, and cattle), the type of home construction (e.g., earthen versus brick walls, thatched versus metal roofs), location (urban 
TABLE 1

Percentage of mortality that occurs in the poorest $20 \%$ of the world's population*

\begin{tabular}{lc}
\hline \multicolumn{1}{c}{ Disease } & \% in lowest quintile \\
\hline Malaria & 58 \\
Diarrheal diseases & 53 \\
Perinatal conditions & 45 \\
Tuberculosis & 44 \\
Maternal conditions & 43 \\
Respiratory infections & 43 \\
HIV/AIDS & 42 \\
\hline * Adapted with the permission of D. Gwatkin. ${ }^{1}$ HIV/AIDS = human immunodeficiency \\
virus/acquired immunodeficiency syndrome.
\end{tabular}

versus rural), and other factors. A score is developed based on the presence or absence of such items. Analyses of differential burden or impact of interventions by wealth status often divide households into quartiles or quintiles based on their asset index.

One major limitation of such indices is that the types of variables and weights given to them vary from country to country, limiting cross-country comparisons. Despite these methodologic limitations, such analyses often yield findings that are consistent from country to country.

Existing studies on the differential burden of malaria and the impact of control interventions by SES and other factors suffer from other limitations. Most studies are analyses of pre-existing data sets which were collected for other purposes. Few studies have been specifically designed to answer questions about malaria burden and the impact of interventions on poor or vulnerable groups. A variety of methodologies for both data collection and analysis have been used, making comparisons of different studies difficult. Notwithstanding these limitations, these studies have yielded some surprising and important results, which are outlined in this report.

\section{RESEARCH FINDINGS}

A small number of studies attempted to describe a relationship between malaria and poverty at the macroeconomic and geographic levels. ${ }^{4}$ The scientific basis of these findings, though, is as yet rather limited.

Of the studies that have examined this issue at the microeconomic level, there have been conflicting findings as to whether malaria incidence differs between the poor and lesspoor. A large sample, multi-country study that analyzed DHS data found little difference in the incidence of fever (as a proxy for malaria) across SES quintiles (Figure 2). ${ }^{5}$ This finding might be explained, though, by the combining of data sets from several countries with different SES characteristics, the lack of specificity of reported fever as a proxy for malaria, and the controlling of several factors that are highly correlated with SES (e.g., housing type and urban versus rural residence). This lack of correlation is supported, though, by evidence from a demographic surveillance site in Tanzania, in which the incidence of fever did not differ significantly across SES quintiles. ${ }^{6}$

In contrast, a few country-specific examinations have identified that the poor may be at greater risk of being infected with malaria. A survey in Zambia, for instance, found a substantially higher prevalence of malaria infection among the population in the lowest wealth quintile (Zambia National Malaria Control Program, unpublished data). A recent review of existing published and unpublished studies related to issues of equity and malaria was not able to draw any conclusions regarding the link between malaria incidence and SES (Worrall $\mathrm{E}$ and others, unpublished data).

Whether the risk of infection varies by SES, current evidence suggests a much stronger correlation between wealth status and the consequences of malaria infection. In rural Tanzania, for instance, mortality in children less than five years old following acute fever was 39\% higher among the poorest compared with the least poor (Mwageni E, unpublished data). The precise reasons for the higher risk of complications from malaria infection in the poor have yet to be elucidated. Many have pointed to the possibility that financial barriers limit access to both preventive and curative services and commodities. Non-financial barriers, including the educational status of the caretaker, distance from health services,

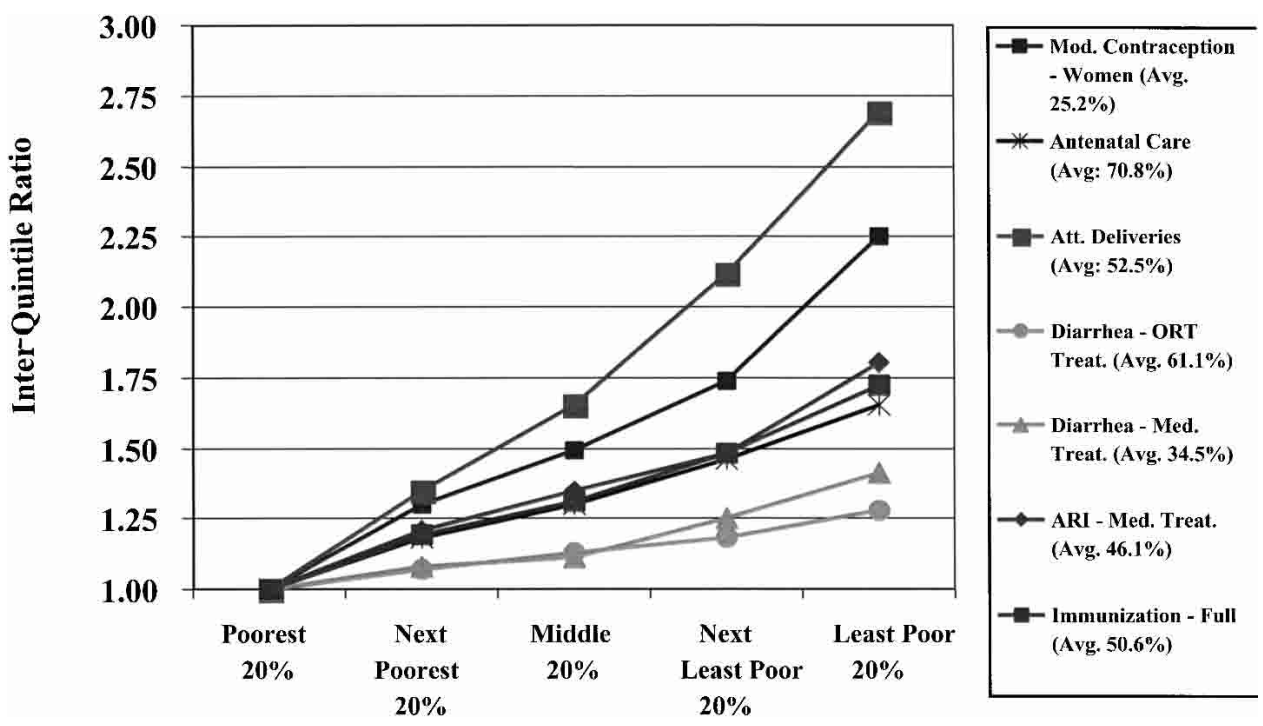

FiguRE 1. Poor-less poor differences in the use of basic health care services in 44 developing countries. Mod. $=$ modern; Avg. $=$ average; Att. $=$ attended; ORT $=$ oral rehydration therapy; Treat. = treated; Med. = medically; ARI $=$ acute respiratory illness. (Used with the permission of D. Gwatkin.) 

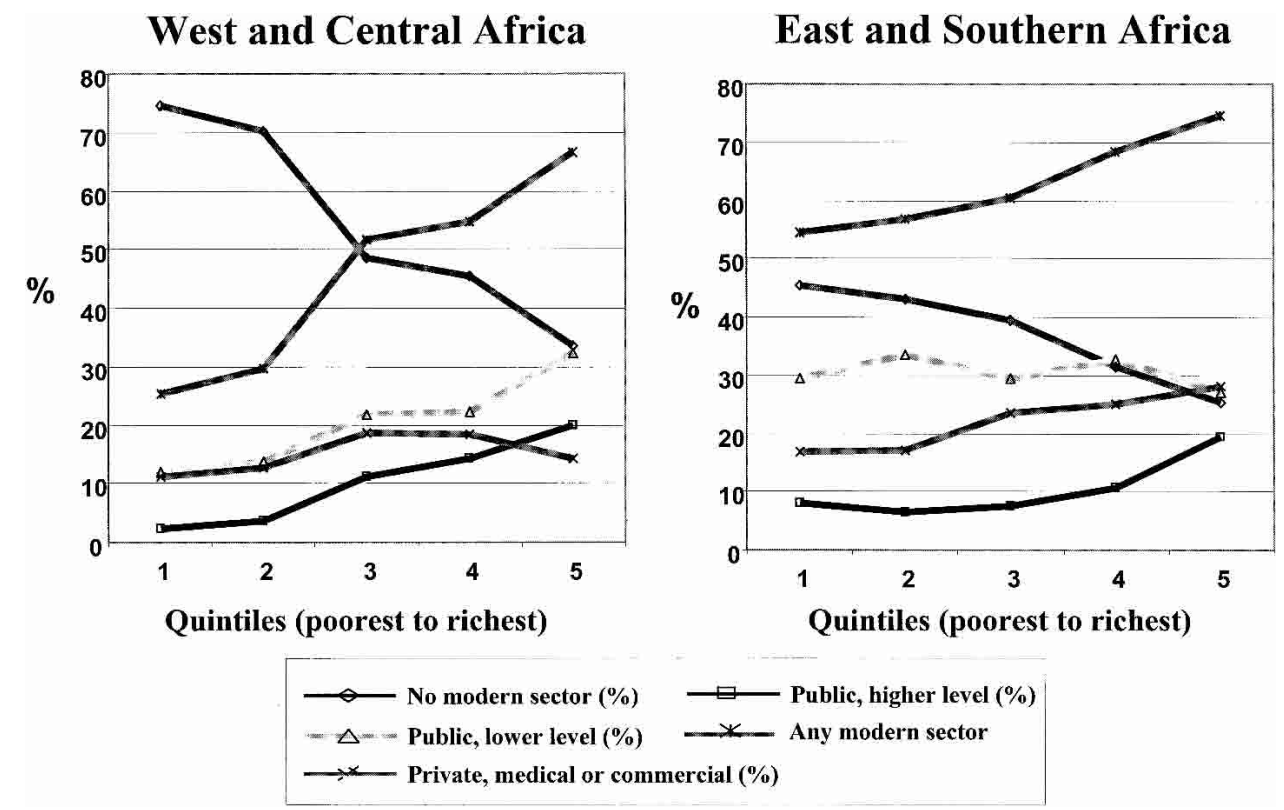

FigurE 2. Source of treatment of fever by socioeconomic quintile in seven West and Central and seven East and Southern African countries. (Adapted with the permission of D. Filmer.)

and opportunity costs of lost time at work, may also be underlying factors.

A number of studies have examined the equity dimensions of the use of preventive measures, particularly ITNs. Data from an ITN social marketing project in Tanzania, for example, demonstrated that the least-poor quartile of the population were 2.74 times more likely to own a bed net than the poorest quartile (Figure 3$)^{7}$

Similar disparities have been found in studies examining access to and use of treatment. Schellenberg and others identified that children less than five years were twice as likely to receive appropriate treatment of fever if their family were in the least-poor quintile than in the poorest quintile $(62 \%$ versus $31 \% ; P=0.0001) .{ }^{6}$ Filmer examined data from DHS surveys in seven eastern and southern African countries and seven western and central African countries to determine differences in treatment seeking for reported fever. ${ }^{5}$ Significant disparities between the poorest and least-poor quintiles were noted in the percentage not receiving any type of formal sector treatment in both eastern and southern Africa (41\% versus $21 \%$ ) and western and central Africa (64\% versus $23 \%$ ).

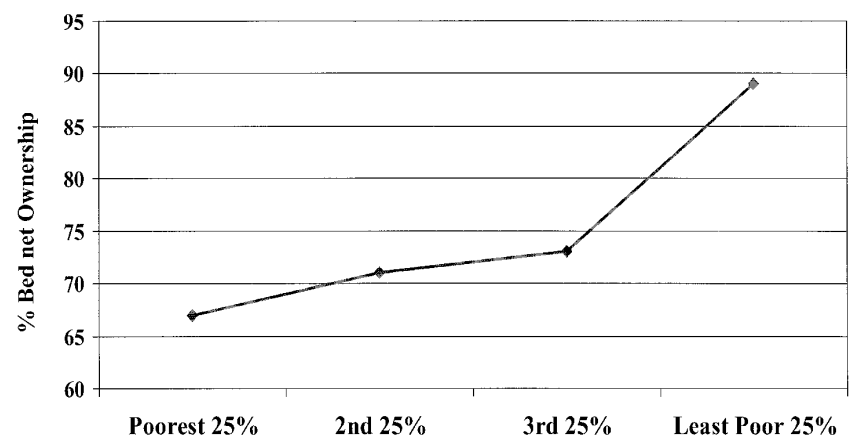

FIGURE 3. Household ownership of bed nets by socioeconomic quartile in southern Tanzania. (Adapted with the permission from S. Abdulla. ${ }^{7}$ )
One factor that no doubt contributes to such disparities is the cost of commodities, such as bed nets and drugs. A study in Malawi found that expenditure on malaria prevention showed a positive correlation with income, indicating that the poorest households probably cannot afford commodities such as ITNs. ${ }^{8}$ Further evidence in Tanzania from an ITN social marketing program supports this argument, finding that the price of the net was the most common constraint on net ownership (Hanson $\mathrm{K}$ and others, unpublished data). This would suggest an important role for targeted subsidies to lower financial barriers to access.

Beyond the costs of commodities, other reasons why the poor have more negative health outcomes and use prevention and treatment less often may be more complex. Cultural, behavioral, and educational factors that lead to delayed treatment seeking may play important roles, but have yet to be fully defined by appropriate qualitative research. Lower levels of education may, for instance, be useful predictors of the type and timeliness of care-seeking behavior. ${ }^{9}$ Elucidation of these factors could suggest interventions for lowering these more complex barriers to the effective use of prevention and treatment services.

\section{DISCUSSION}

Clearly in this new line of investigation, there is much that we do not know. For example, studies examining the relationship between malaria incidence and SES have yielded contradictory results. Filmer found no positive correlation between reported fever and SES in his analysis of DHS data. ${ }^{5}$ Other studies have contradicted these findings.

In contrast, some noticeable trends can be detected in studies looking at severe complications of malaria, and these findings could inform the implementation of malaria control programs. There appears to be a much stronger basis to conclude that the severe consequences of malaria are borne most heavily by the poorest. More limited access to both preventive 
measures and curative treatment may partially explain worse outcomes among the poorest.

Not surprisingly, studies looking at both use of ITNs and access and use of malaria treatment demonstrate lower coverage in the poorest compared with the least-poor. It remains to be clarified whether the barriers to preventive and treatment services are primarily financial or whether other factors (e.g., cultural practices and norms, sex roles, caretakers' educational status, proximity to health services) play a significant role. Disentangling the myriad factors that might limit the accessibility and use of malaria control services by the poor will require additional quantitative and more importantly qualitative research.

\section{REACHING THE POOR WITH MALARIA CONTROL INTERVENTIONS}

Within the RBM partnership, all agree that there is a pressing need to increase coverage of ITNs and drug treatment in the poorest. In general terms, lowering the price of ITNs and treatment, either through reducing production costs, elimination of taxes, and tariffs, or providing subsidies to consumers, will almost certainly result in increases in coverage. There has been debate, though, about the precise methods by which to achieve such cost reductions and increases in coverage. Different approaches have been tried for increasing the coverage of ITNs, including public sector distribution, social marketing by non-government organizations, and public-private partnerships with sale through commercial outlets. Often these approaches include the use of universal or targeted subsidies, either through sale of subsidized products or the use of vouchers. To date, there has been no definitive evidence indicating that any of these approaches is more or less effective in reaching the most poor.

Similarly, various approaches are being promoted to increase the use of highly effective drug treatment of symptomatic malaria, either through public sector facilities, community outreach, or the private sector. Evidence on the use of key public health services suggests that providing treatment only through public health facilities may fail to reach the poor. Little is known, though, about alternative approaches. Will the poor be more effectively reached by providing treatment through community-level volunteers or by training private sector providers and drug sellers to provide the appropriate drug and dose to persons with malaria? Determining how coverage in the poor can most efficiently and effectively be increased must be a high priority if the goals of RBM are to be achieved.

Unfortunately, existing information on disparities in burden of malaria and coverage of key malaria control interventions raises more questions than it yields answers to these questions. Even in those areas where we can draw some conclusions, such as the increased risk of complications among the poorest, we can only hypothesize about the reasons why such disparities exist.

With this in mind, a brainstorming meeting was held in September 2002, sponsored by the London School of Hygiene and Tropical Medicine and the World Bank on behalf of the RBM Partnership. Experts in malaria control, monitoring and evaluation, economics, and program financing and implementation were brought together to review current information on equity and malaria, make recommendations on strategies for going to scale with malaria control interventions, and identify areas for further investigation.

This panel recommended that the RBM partnership should include considerations of equity within its goals and objectives for malaria control. Monitoring and evaluation to assess progress towards the goals of RBM at both the global and country level should incorporate measurements of SES, sex, and possibly other factors (e.g., educational status of caretakers) into their data collection and analysis schemes.

The group also noted that studies were now underway to assess the effectiveness of various strategies to reach the poor within other health and non-health related interventions. Such interventions in malaria control should be informed by these experiences.

The group expressed the need for additional research that was designed with issues of equity in mind. It noted that malaria-affected countries were using a diversity of approaches for going to scale with malaria control interventions. This would provide ample opportunities to examine which of these approaches most effectively reached the poor.

There was also acknowledgment that while such original research was being carried out, further analysis of existing data sets could begin to provide some clues to what types of interventions are likely to reach the most poor. In particular, data from DHS, the United Nations Children's Fund (UNICEF) Multiple Indicator Cluster Surveys (MICS), and ongoing demographic surveillance sites (e.g., INDEPTH network) provide opportunities to begin to develop a better understanding of existing inequities and possibly provide insights into how the poorest can be reached.

Furthermore, examination of equity should not only be limited to SES, but rather look comprehensively at various other factors that might have an equal or greater impact on morbidity and the use of prevention and treatment services. These should include sex, age, marital and educational status, occupation, and other cultural and behavioral factors.

Among the priority areas for further study should be the effectiveness of vouchers or other methods for providing targeted subsidies for essential commodities (e.g., ITNs and drugs) to improve access to the poorest. The need to assess factors that influence demand and supply for malaria control measures was also highlighted.

Lastly, and not specific to malaria research per se, there is a need for continuous improvement of methods for measuring SES, as well as increased acknowledgment of the more complex cultural and social factors that might lead to inequities. However, it is heartening to observe that even with the imperfect nature of current measures of SES, much has already been learned that will help guide program implementation and future research.

Received for publication August 21, 2003. Accepted for publication January 6, 2004.

Acknowledgements: This paper is based on a review and analysis of the literature and a report on a meeting on Malaria and Equity carried out by the Health Economics and Financing Program, London School of Financing and Tropical Medicine, in collaboration with the World Bank, and funded by the World Bank.

Financial support: Natasha Palmer, Eve Worrall, Kara Hanson, and Anne Mills are members of the Health Economics and Financing Programme, which receives a research grant from the United Kingdom Department for International Development. 
Authors' addresses: Lawrence M. Barat, Academy for Education Development, 1825 Connecticut Avenue, Washington, DC 20009 and Suprotik Basu, Natasha Palmer, Eve Worrall, Kara Hanson, and Anne Mills, Health Economics and Financing Programme, London School of Hygiene and Tropical Medicine, Keppel Street, London WC1E 7HT, United Kingdom.

\section{REFERENCES}

1. Gwatkin DR, Guillot M, 2000. The Burden of Disease among the Global Poor: Current Situation, Future Trends, and Implications for Strategy. Geneva: Global Forum for Health Research Publications.

2. Castro-Leal F, Dayton J, Demery L, Mehra K, 1999. Public social spending in Africa: do the poor benefit? World Bank Res Observer 14: 49-72.

3. Whitehead M, 1992. The concepts and principles of equity and health. Int J Health Services 22: 429-445.
4. Sachs J, Malaney P, 2002. The economic and social burden of malaria. Nature 415: 680-685.

5. Filmer D, 2002. Fever and Its Treatment in the More and Less Poor in Sub-Saharan Africa. Washington, DC: World Bank. World Bank Policy Research Working Paper \#WPS2789.

6. Schellenberg JA, Victoria CG, Mushi D, de Savigny D, Schellenberg D, Mshinda H, Bryce J, 2003. Inequities among the very poor: Health care for children in rural southern Tanzania. Lancet 361: 561-566.

7. Abdulla S, Schellenberg JA, Nathan R, Mukasa O, Marchant T, Smith T, Tanner M, Lengeler C, 2001. Impact on malaria morbidity of a programme supplying insecticide treated nets in children aged under 2 years in Tanzania: Community cross sectional study. BMJ 322: 270-273.

8. Ettling M, 1994. Economic impact of malaria in Malawian households. Trop Med Parasitol 45: 74-79.

9. Fawole OI, Oneadeko MO, 2001. Knowledge and home management of malaria fever by mothers and care givers of under five children. West Afr J Med 20: 152-157. 